\title{
Numerical Simulation of Zinc Flow in Different Layouts of Galvanization Pot
}

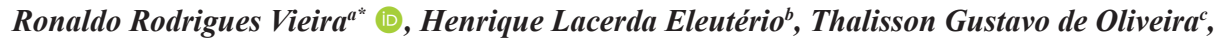 \\ Maurício Covcevich Bagatini ${ }^{d}$, Roberto Parreiras Tavares ${ }^{d}$ \\ a Unigal Usiminas, Avenida Pedro Linhares Gomes, 5431, Ipatinga, MG, Brasil \\ ${ }^{b}$ Centro de Pesquisa e Desenvolvimento da Usiminas, Avenida Pedro Linhares Gomes, 5431, \\ Ipatinga, $M G$, Brasil \\ 'Instituto ESSS, Rua Orlando Phillippi, 100, Florianópolis, SC, Brasil \\ 'Universidade Federal de Minas Gerais, Departamento de Metalurgia, Avenida Antonio Carlos, 6627, \\ 31270-900, Belo Horizonte, MG, Brasil
}

Received: October 14, 2020; Revised: October 26, 2020; Accepted: February 15, 2021

\begin{abstract}
The production of galvanized steel with zinc (GI) presents great challenges due to the high demand for surface quality required for its applications. The automotive segment is one of the largest consumers of this type of product. In hot dip galvanizing lines, the main defect that affects the surface quality of the coatings is the dragging of dross particles. Some studies have already been carried out to understand the formation of these particles inside the zinc bath, however little is known about the trajectory of these particles in relation to the pot layout. In this study, two types of layout were simulated to assess how this interferes with the flow of zinc. In the results it was verified that, the position of the heating inductor significantly modifies the trajectory of the dross particles.
\end{abstract}

Keywords: Numerical Simulation, Mathematical Model, Hot Dip Galvanizing, Dross.

\section{Introduction}

The hot dip galvanizing process is used to produce coatings on carbon steel substrates, to increase corrosion resistance. These zinc-coated carbon steel materials are intended for various sectors such as appliance manufacturing, construction and especially for the automotive industry. Among the materials supplied to the automotive industry some will compose the internal panel and those that will be used as exposed panels. In the exposed panels, the degree of demand for superficial aesthetic quality is very high, therefore, the rigor of control of the process parameters of these materials must be very strict, as defects that may be apparent on the surfaces of the materials would make them unsuitable for use in exposed panels.

Dross dragging is the main and best-known defect that can occur on the surface of the galvanized sheet during the coating process. The particles of dross entrained by the strip may have various shapes and dimensions and can have found the strip in different positions inside the zinc bath. In recent years, some studies have been developed to understand the phenomena that involve the generation of dross particles inside the galvanizing pot, which are combinations between the Fe provided by the steel strip in process with the $\mathrm{Al}$ and $\mathrm{Zn}$ that make up the galvanizing bath. Some studies have investigated the effects of the inlet temperature of the steel strip in the zinc bath in the greater dross formation ${ }^{1,2}$ proposing the control of this parameter and with good results in reducing the formation of dross particles, others were conducted with thermodynamic analyzes formation of these particles, finding

*e-mail: ronaldo.rodrigues@unigalusiminas.com the effect of the relationship between temperature and the solubility of $\mathrm{Fe}$ and $\mathrm{Al}$ in the zinc bath in this formation ${ }^{3,4}$ also with good results in reducing the formation of these particles. The analysis of the flow of zinc in the bath for a particular zinc pot layout was also investigated, where it was proposed to place barriers to prevent the formation of vortexes ${ }^{5}$ and as a result the number of particles dragged by the strip was reduced.

These studies were developed in numerical models of simulation software, usually for this kind of simulation Ansys CFX or Fluent using the finite volume method for modeling and calculation are the most used. These models represent well the process of industrial conditions and facilitated the understanding of the phenomena involved in the process of electroplating without the need for realization of industrial tests, which could put at risk the production, quality and operational cost.

As is already known, it is not possible to prevent the formation of dross in the zinc pot, but only to control it ${ }^{6}$. Knowing the path they take when they are dragged by the movement of the zinc bath can help prevent them from finding the surface of the strip and end up causing surface defects, in these studies already mentioned, the simulations were developed in a pot model with side inductors in regarding the position of the strip. However, there are other types of construction of pots with inductors in other positions and there is the possibility of the flow of zinc by these inductors in different positions to change the trajectories of the particles within the zinc pot.

The objective of this study is to measure exactly these possible differences simulating two types of pot layouts, 
one with side inductors over the strip, named in this project type A pot, as previously done in the studies mentioned, and the other with front inductors to the strip, called in this project type B pot and compare the results.

\section{Experimental}

In this present study, the top-dross particle displacement directions $\left(\mathrm{Fe}_{2} \mathrm{Al}_{5} \mathrm{Zn}_{\mathrm{x}}\right)^{7}$ were simulated in a 3D computational model in two $\mathrm{Zn}$ pot configurations, Figure 1. The first configuration presents the side inductors to the strip and, the second presents the frontal inductors. The destination of the dross particles was mapped and recorded.

To perform the simulations, ANSYS Fluent software was used, with the $\mathrm{k}-\varepsilon$ Realizable turbulence model, which uses the equation for turbulent viscosity $\mu_{t}$.

$\mu_{t}=\rho C_{\mu} \frac{k^{2}}{\varepsilon}$

Where $k$ is the turbulent kinetic energy, $\varepsilon$ is the rate of turbulent dissipation and $\rho$ is the fluid density. This model differs from $k-\varepsilon$ Standard exactly in the term $C_{\mu}$, which was constant in the standard model, and is now a function of turbulence, deformation rates and average flow rotation ${ }^{8}$.

The results of the flow lines of pot A were compared with the results already known in the literature to confirm the flow pattern ${ }^{9,10}$. The results of pot B were compared with those of pot A and the differences were mapped.

To trace the direction lines of the particles, the one-way Discrete Phase Model (DPM), also called the Lagrangian model, was used. It takes into account the forces of liquid zinc, resulting from buoyancy and viscosity acting on the particles, but does not compute the forces of the particle on zinc, as they were considered as negligible mass concerning the mass of the zinc flow, since the particle size in the bath, its contributions to the flow are negligible. In this model, the $2 \mathrm{~s}$ time step was used with 10 interactions per time jump and the maximum number of 50,000 steps for calculating the trajectory of the particles.

To carry out the simulations, the following flow conditions for molten zinc were defined:

a) Molten zinc is an incompressible liquid and behaved like a Newtonian fluid;

b) The flow of liquid zinc in the bath is described by the Navier-Stokes equations adapted to the turbulent flow;

$\rho \frac{D u}{D t}=-\nabla \rho+\nabla[2(\mu+\mu t) \dot{\gamma}(\mu)]-\rho g$

$\nabla u=0$

Where: $\dot{\gamma}$ is the fluid deformation rate.

c) The flow regime is permanent, turbulent and the equations that govern the kinetic energy of the turbulence and the dissipation rate of this energy are;

$\rho \frac{D k}{D t}=\nabla \cdot\left[\left(\mu+\frac{\mu_{T}}{\sigma_{k}}\right) \nabla k\right]+P+G-\rho \varepsilon$

$\rho \frac{D \varepsilon}{D t}=\nabla \cdot\left[\left(\mu+\frac{\mu_{T}}{\sigma_{\varepsilon}}\right) \nabla \varepsilon\right]+C \varepsilon l \frac{\varepsilon}{k}(P+G)-C_{\varepsilon 2 \rho} \frac{\varepsilon^{2}}{k}$

d) The finite volume numerical method used the SIMPLE ${ }^{10,11}$ algorithm to solve the equations performed;

e) The selection method was based on the methods most commonly used in publications ${ }^{10,11}$, the pressure was discretized with the standard scheme, while momentum, turbulent kinetic energy, turbulent

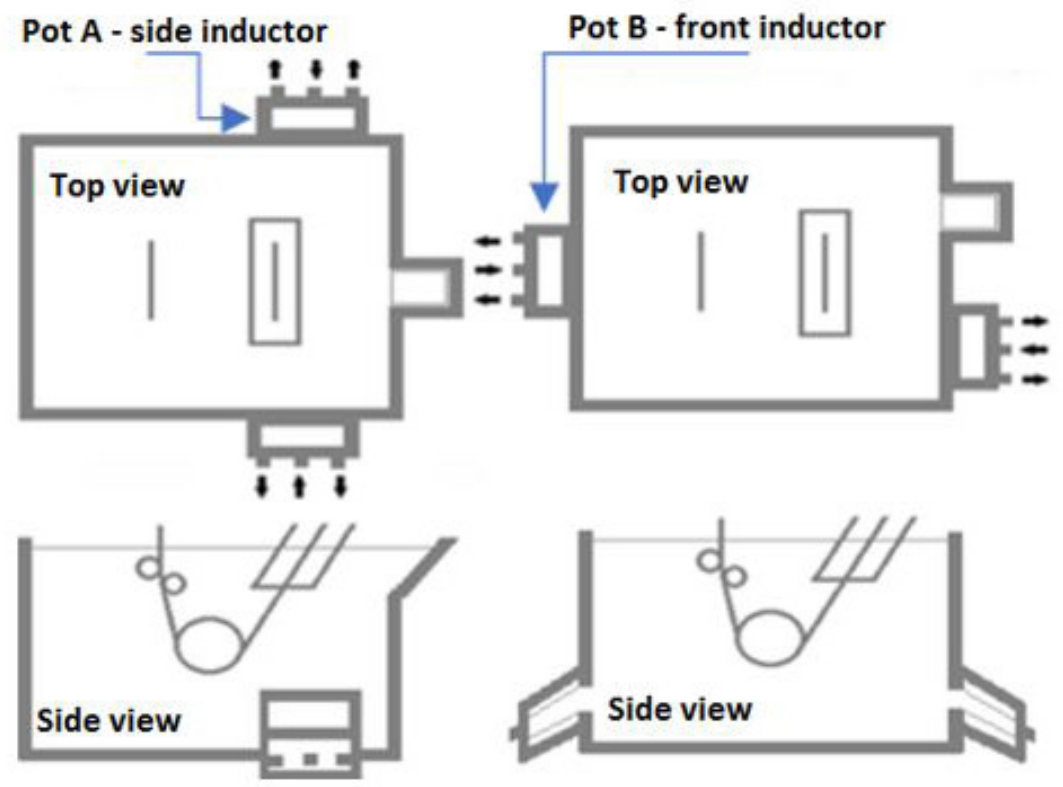

Figure 1. Top and side view of the galvanizing pots with different positions of electric inductors. Reference: Paper authors. 
dissipation rate and equations of energy are discretized with the first-order upwind scheme ${ }^{11,12,13}$;

f) The convergence criteria for calculations of momentum, velocities, turbulence and dissipation of turbulence energy were the results of the residuals for calculating interactions less than $1 \times 10^{-5}$.

The following conditions were used for the simulation according to Table 1 and items of boundary conditions:

- Constant temperature and volume;

- $\quad$ Zinc speed in the inductors $2 \mathrm{~m} \mathrm{~s}^{-1} 14,15$;

- $\quad$ Strip speed in process $2.5 \mathrm{~m} \mathrm{~s}^{-1}$;

- Top-dross particles with diameters of $20 \mu \mathrm{m}$ and $250 \mu \mathrm{m}$ injected in "V zone";

- $\quad$ Top-dross particles with a diameter of $500 \mu \mathrm{m}$ and $1000 \mu \mathrm{m}$ randomly injected into the zinc bath;

- There is no relative movement between the strip and the submerged rollers;

- Top-dross particle injection rate of $4.33 \times 10^{-4} \mathrm{~kg} \mathrm{~s}^{-1}{ }^{16}$;

- The surface of the zinc bath defined as the top-dross removal region;

- $\quad$ Strip surface defined as the top-dross trapping region;

- Top-dross adhesion on the roller surface disregarded;

- Adhesion of top-dross on the walls of the pot and the structure of the roller arms disregarded;

- The strip was divided into 4 regions, Figure 2, to better map the amount of top-dross particles carried to each region of the strip by the flow of zinc, in Figure 2 the stabilizer and deflector rollers that are submerged in the zinc bath, there is still the snout region and the submerged rolls.

The models of pots A and B tested have the inductors in the following positions identified by the dimensions $\mathrm{H1}=900 \mathrm{~mm}$; $\mathrm{H} 2=1795 \mathrm{~mm} ; \mathrm{H} 3=3900 \mathrm{~mm} ; \mathrm{H} 4=1320 \mathrm{~mm}$ and $\alpha=60^{\circ}$ ( $\mathrm{H} 4$ and $\alpha$ are common for pots $\mathrm{A}$ and $\mathrm{B}$ ) and $\mathrm{H} 5=1500 \mathrm{~mm}$, according to Figures 3, 4 and 5 .

\section{Results and Discussion}

During the simulations, a mesh independence test was performed, varying the maximum size of the volume element from $320 \mathrm{~mm}$ to $20 \mathrm{~mm}$, and for sizes less than $40 \mathrm{~mm}$, no variations in flow directions and speeds were observed.

Performing the simulation with the two types of pots, the mesh of the type A pot consisted of 1,692,309 volume elements formed by $6,335,290$ nodes, whereas the type B pot, the mesh had 1,661,580 volume elements formed by $6,211.058$ nodes. For both cases, the type of element used was polyhedral.

For the comparison of the flow pattern results obtained in the two types of pots, frontal cut plans were plotted in the two types of pots in significant regions in relation to their construction differences, these plans are shown in Figures 6 to 7 and the differences observed, identified and commented below.

Comparing the flow profile of the two types of pot configuration, it can be seen that pot A, with side inductors, Figure 6, shows a flow of zinc from the inductors towards the bottom of the strip (region 1 and 2). In the region where the strip enters the bath, vortexes are formed below the crossing of the flows (regions 3 and 4). In Figure 7, referring to pot $\mathrm{B}$, there is an upward flow zone from the
Table 1. Properties of top-dross particles and molten zinc.

\begin{tabular}{ccc}
\hline Property & Value & Unit \\
\hline Temperature of top-dross & 460 & ${ }^{\circ} \mathrm{C}$ \\
\hline Density ${ }^{12)}$ of top-dross & 4200 & $\mathrm{~kg} \mathrm{~m}^{-3}$ \\
\hline Zinc temperature & 460 & ${ }^{\circ} \mathrm{C}$ \\
\hline Zinc $^{12)}$ density & 6700 & $\mathrm{~kg} \mathrm{~m}^{-3}$ \\
\hline Zinc $^{13)}$ viscosity & $3.85 \times 10^{-3}$ & $\mathrm{~Pa} \mathrm{~s}$ \\
\hline
\end{tabular}

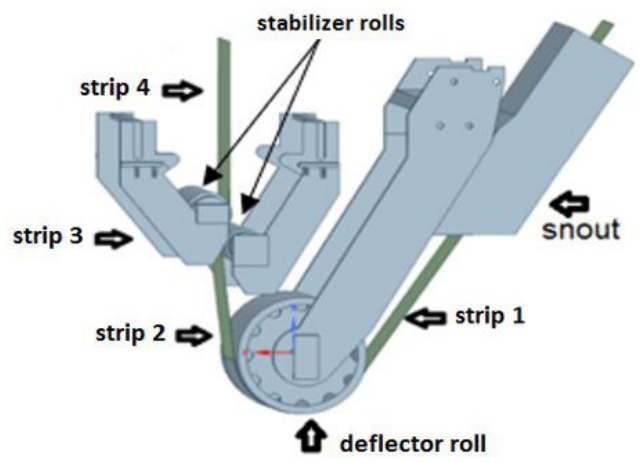

Figure 2. Definition of the regions inside the zinc bath. Reference: Paper authors.

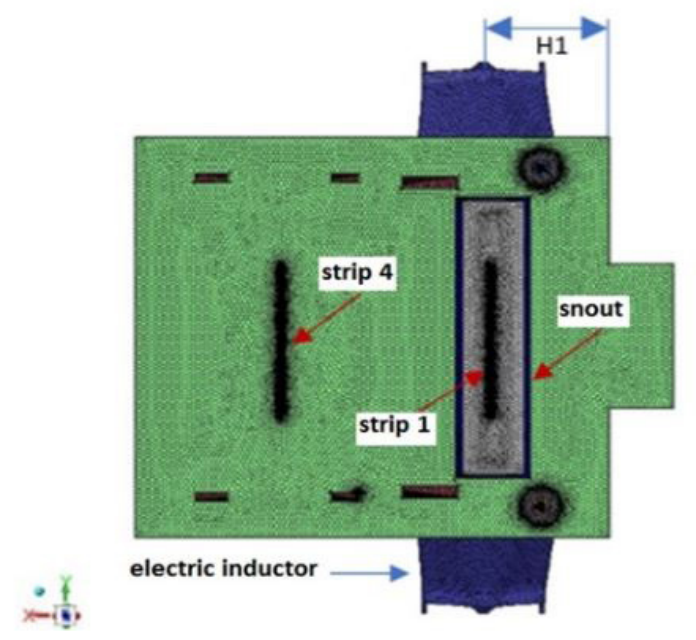

Figure 3. Top view of pot A with the horizontal positioning of the side inductors on the strip. Reference: Paper authors.

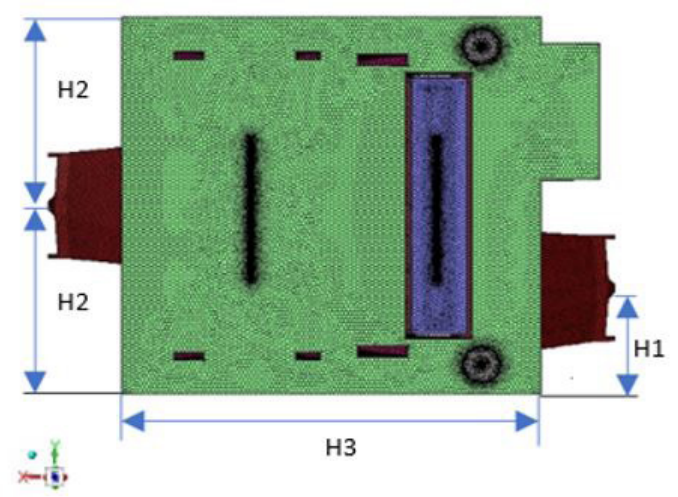

Figure 4. Top view of pot B with the horizontal positioning of the inductors in front of the strip. Reference: Paper authors. 


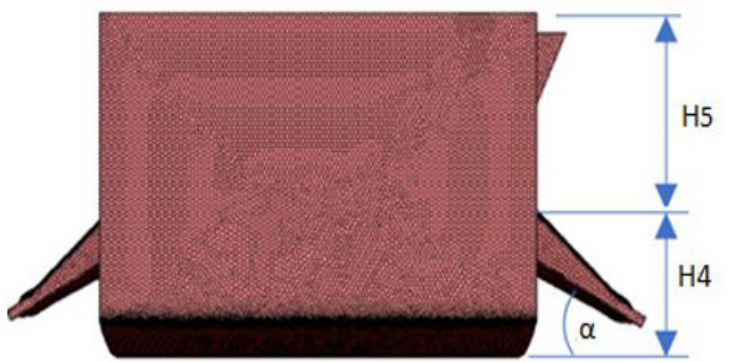

$\frac{i}{x \rightarrow(0)}$

Figure 5. Side view of pot B with the vertical positioning of the inductors in front of the strip. Reference: Paper authors.

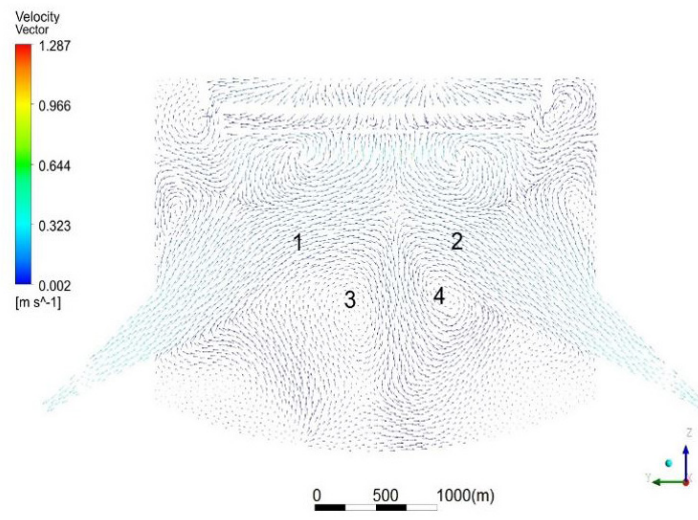

Figure 6. Front view of pot A with inductors lateral to the strip with flow pattern drawn by velocity vectors. Reference: Paper authors. Analysis performed during this research.
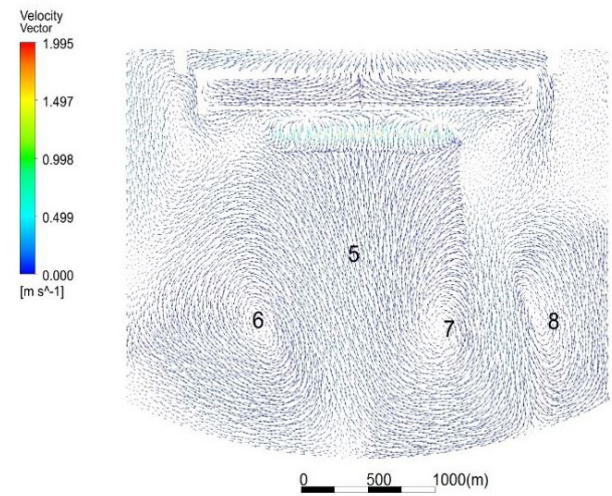

Figure 7. Front view of pot B with inductors in front of the strip with a flow pattern drawn by velocity vectors. Reference: Paper authors. Analysis performed during this research.

bottom of the pot to the bottom of the region where the strip enters the bath (region 5). Unlike pot A, where the regions with vortex formation $(6,7$, and 8$)$ are closer to the bottom of the pot. These differences observed in the flow of zinc can be attributed to the effect of the only difference between the two types of pot, which is the position of the electric heating inductors of the zinc bath.

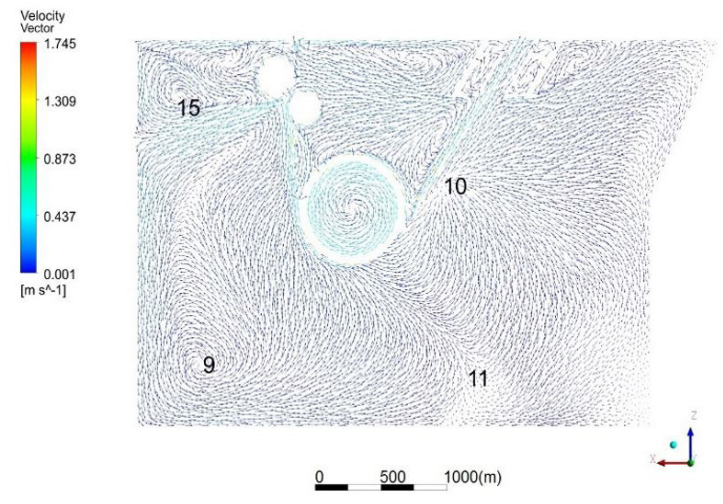

Figure 8. Side view of pot A with inductors lateral to the strip with a flow pattern drawn by velocity vectors. Reference: Paper authors. Analysis performed during this research.

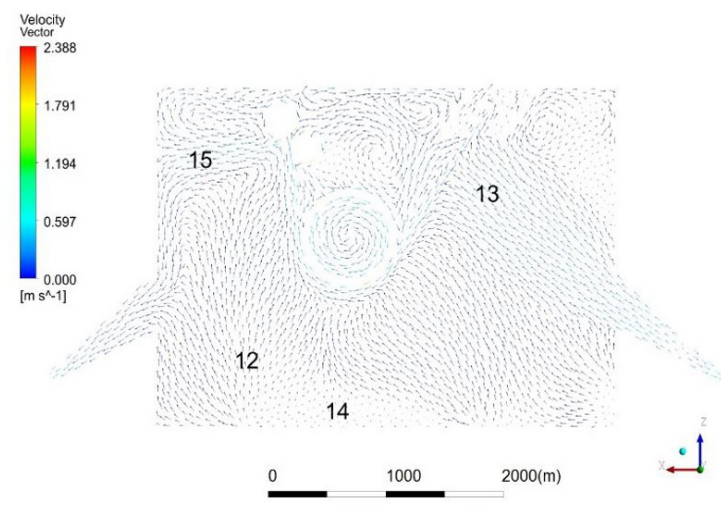

Figure 9. Side view of pot B with inductors in front of the strip with a flow pattern drawn by velocity vectors. Reference: Paper authors. Analysis performed during this research.

Figures 8 to 9 shows the differences observed in the comparison of the flow pattern results obtained in lateral planes plotted in the two types of pots again in significant regions in relation to their constructive differences. Comparing Figures 8 and 9 , with the images obtained by observing the lateral plane plotted in the middle of pots A and B, it appears that the regions 9 of pot $\mathrm{A}$ and 12 of Pot $\mathrm{B}$, despite being in the same position, have profiling different flows. In region 9 , there are vortexes, while in region 12 there is an upward flow. In the comparison between regions 10 and 13, it can be seen that in pot A the flow vectors are orthogonal to the observation point, with a small portion being directed into the snout, and in pot $\mathrm{B}$, the vectors are parallel to the observation and much of it being directed to the strip and into the snout. In regions 11 and 14, there is a flow parallel to the observation point in pot $\mathrm{A}$, while in pot $\mathrm{B}$, the flow is mostly orthogonal. As already mentioned, the only difference between the two types of simulated pots is the position of the inductors, the flow coming from the inductors, as it can be seen, interacts with the flow lines from the movement of the strip and the submerged rolls in the zinc, altering significantly the flow pattern of one type of pot concerning to the other. In this comparison, it is possible to verify that the only region where the flow pattern is repeated from one 
pot to the other is identified as region 15 , where there is a formation of a small vortex caused by the interaction of the flow of zinc from one of the stabilizing rollers with the flow of zinc around it.

In the simulation of the displacement of the top-dross particles using the Lagrangian method it was possible to verify that, although the particles have a density much lower than the bath, they move throughout the volume of zinc. They were dragged by the flow currents produced by the movement of the strip, rollers and electrical inductors. Particles with larger diameters $(500 \mu \mathrm{m}$ and $1000 \mu \mathrm{m})$ precipitate on the bath surface more quickly, while smaller particles $(20 \mu \mathrm{m}$ and $250 \mu \mathrm{m})$ spend more time moving in the zinc bath until they find the surface. This difference in behavior between the flow of particles is explained by the buoyant force that acts more significantly on bodies of greater volume when compared to those of less volume and the same density. In Figures 10, 11, 12 and 13 the paths are traced by the particles of $20 \mu \mathrm{m}$ and $500 \mu \mathrm{m}$ until they are dragged by the strip or precipitate on the surface of the bath. The $20 \mu \mathrm{m}$ particles were injected into the model in the region known as "V zone" because this region is outlined by the strip and, consequently, has a greater presence of Fe to start the formation of top-dross particles. The $500 \mu \mathrm{m}$ ones were dispersed in the interior of the bath since in this dimension they are already free from vortexes and travel through the zinc bath.

Looking at Figures 10 and 11, it can be seen that the $20 \mu \mathrm{m}$ particles have more sinuous paths in pot A than the $500 \mu \mathrm{m}$ particles, as they are more affected by the vortexes present in the bath. As for the $500 \mu \mathrm{m}$ particles, the paths drawn are more vertical and less sinuous. The same can be seen in pot B, the smaller particles travel longer paths inside the bath, increasing their residence time, Figures 12 and 13 .

Comparing the two pot configurations, it can be seen that, especially for the $20 \mu \mathrm{m}$ particles, in pot A there are less sinuous paths traced than in pot B. Now for $500 \mu \mathrm{m}$ particles, this difference, despite existing, is not so evident.

Some studies suggest that dross particles are formed and coalesce within the vortexes within the bath ${ }^{16}$. These particles, when they reach a size greater than $20 \mu \mathrm{m}$, leave these vortices and pass through the bath until they are dragged by the strip ${ }^{16}$. In Tables 2 and 3 it is possible to check the destination of particles of different diameters in the two types of pots. It is possible to notice in both configurations that the larger diameter particles precipitate on the surface of the bath in greater numbers while the particles with smaller diameters find the strip inside the bath in a more representative way, increasing the risk of being dragged by the strip. Another fact that can be clearly perceived is the greater number of larger particles of top-dross precipitated on the surface of the bath that is inside the snout of pot B when compared to that of $\mathrm{A}$. The precipitation of larger particles in this region can represent an accumulation of top-dross very close to that of the strip and, consequently, will result in the dragging of these particles.

Still analyzing the data in Tables 2 and 3, it can be seen that the amount of top-dross particles that found the strip in region 1 , in the configuration of pot $\mathrm{A}$, is higher than the amount of particles that find the strip in the same region of

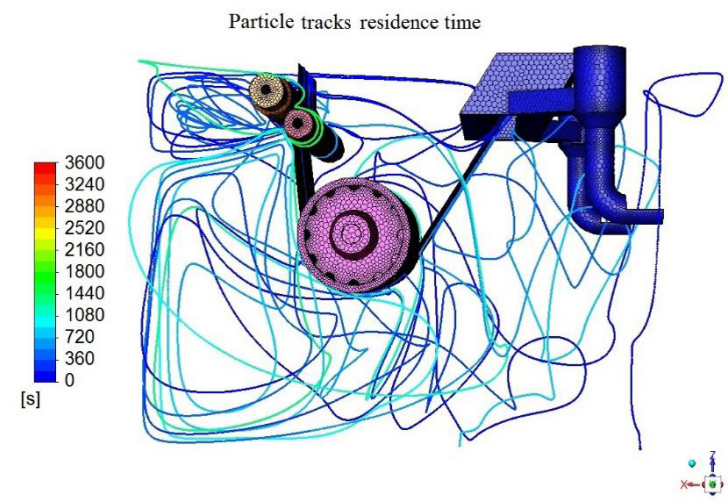

Figure 10. Residence time of $20 \mu \mathrm{m}$ top-dross particles in the zinc bath and the path traced by them inside the type A pot. Reference: Paper authors. Analysis performed during this research.

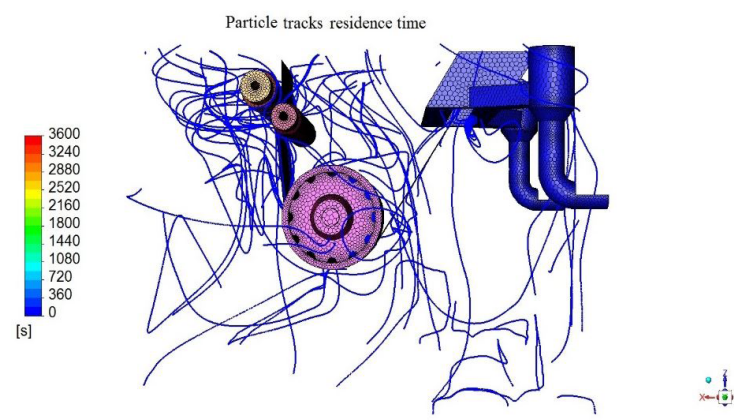

Figure 11. Residence time of $500 \mu \mathrm{m}$ top-dross particles in the zinc bath and the path traced by them inside the type A pot. Reference: Paper authors. Analysis performed during this research.

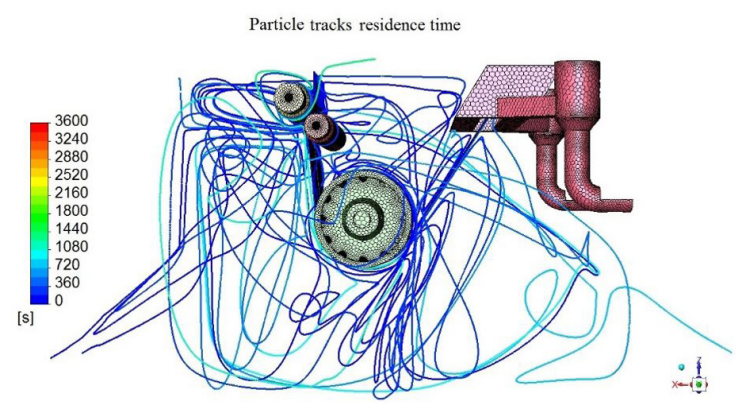

Figure 12. Residence time of $20 \mu \mathrm{m}$ top-dross particles in the zinc bath and the path traced by them inside the type B pot. Reference: Paper authors. Analysis performed during this research.

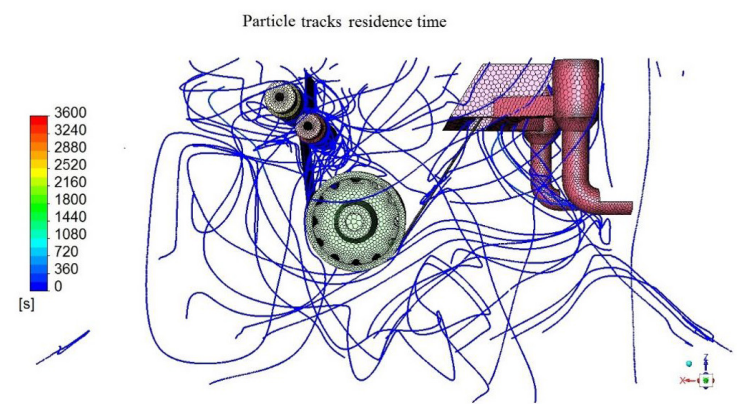

Figure 13. Residence time of $500 \mu \mathrm{m}$ top-dross particles in the zinc bath and the path traced by them inside the type B pot. Reference: Paper authors. Analysis performed during this research. 
Table 2. Destination of top-dross particles simulating $1 \mathrm{~h}$ of process in Pot A.

\begin{tabular}{|c|c|c|c|c|c|c|c|}
\hline \multirow{2}{*}{$\mu \mathrm{m}$ particle } & \multirow{2}{*}{ Total } & \multicolumn{2}{|c|}{ Precipitated in } & \multicolumn{3}{|c|}{ Found the } & \multirow{2}{*}{ Still in transit } \\
\hline & & Bath surface & Bath surface in snout & strip 1 & strip 2 & strip 3 & \\
\hline 20 & 610 & $18 \%$ & $0 \%$ & $41 \%$ & $21 \%$ & $14 \%$ & $6 \%$ \\
\hline 250 & 610 & $29 \%$ & $4 \%$ & $26 \%$ & $21 \%$ & $14 \%$ & $7 \%$ \\
\hline 500 & 876 & $63 \%$ & $6 \%$ & $8 \%$ & $1 \%$ & $1 \%$ & $22 \%$ \\
\hline 1000 & 876 & $61 \%$ & $7 \%$ & $8 \%$ & $2 \%$ & $1 \%$ & $21 \%$ \\
\hline
\end{tabular}

Reference: Paper authors. Analysis performed during this research

Table 3. Destination of top-dross joints simulating $1 \mathrm{~h}$ of the process in Pot B.

\begin{tabular}{|c|c|c|c|c|c|c|c|}
\hline \multirow{2}{*}{$\mu \mathrm{m}$ particle } & \multirow{2}{*}{ Total } & \multicolumn{2}{|c|}{ Precipitated in } & \multicolumn{3}{|c|}{ Found the } & \multirow{2}{*}{ Still in transit } \\
\hline & & Bath surface & Bath surface in snout & strip 1 & strip 2 & strip 3 & \\
\hline 20 & 567 & $18 \%$ & $0 \%$ & $40 \%$ & $23 \%$ & $16 \%$ & $3 \%$ \\
\hline 250 & 567 & $28 \%$ & $0 \%$ & $32 \%$ & $23 \%$ & $16 \%$ & $2 \%$ \\
\hline 500 & 866 & $56 \%$ & $10 \%$ & $4 \%$ & $1 \%$ & $1 \%$ & $28 \%$ \\
\hline 1000 & 866 & $62 \%$ & $12 \%$ & $3 \%$ & $3 \%$ & $1 \%$ & $18 \%$ \\
\hline
\end{tabular}

Reference: Paper authors. Analysis performed during this research.

the pot B. This fact can also represent a potential risk of dross dragging since the strip in region 1 is anterior to the submerged deflecting roller, which means that if the particles are fixed on the surface of the material in the process by this roller, they will certainly be dragged giving rise to the dross drag defect.

The strip in region 4, as it has an area of exposure to the flow of zinc smaller than the other regions, has no longer had a submerged roller and is about to leave the bath with the reactions that form the finished coating ${ }^{17,18}$, it was not considered as capable of dragging particles and for that reason, it is not in Tables 2 and 3. The other regions of the strip in the configuration of pot A have, on average, more chance of dragging top-dross particles than in the configuration of B.

\section{Conclusion}

Using a computer simulation of the fluid dynamics flow of the dross particles with the ANSYS Fluent software, it was possible to verify that the position of the electric heating inductors significantly alters the flow of liquid zinc inside the pot, changing the vortex formation position and the path taken by top-dross particles. It has been found that larger particles are easier to precipitate on the bath surface than smaller particles.

It was possible to verify that in pot A there is a potential increase in the risk of the strip dragging dross particles fixed on its surface by the submerged deflector roller and also in the other regions of the strip, which possibly can be changed by altering the distance between the output of the inductors and the strip submerged in the bath, in pot B, a potential increase in the risk of precipitation of top-dross inside the snout was identified, which may be a greater risk since the accumulation of dross inside the snout could prevent the production of materials of any level of superficial quality requirements.

\section{References}

1. Kim YH, Cho YW, Chung SH, Shim JD, Ra HY. Numerical analysis of fluid flow and heat transfer in molten zinc pot of continuous hot-dip galvanizing line. ISIJ Int. 2000;40:706-12.
2. Yu KR, Ilinca F, Goodwin FE. Numerical simulation of the effect of strip entry temperature on continuous galvanizing bath management and dross formation. In: The Iron and Steel Technology Conference, AISTech; 2016 may 16-19; Pittsburgh, PA. Proceedings. Pittsburgh, PA: Association for Iron and Steel Technology; 2016. p. 2043-53.

3. Ilinca F, Hétu JF, Ajersch F. Numerical simulation of 3D turbulent flow and heat transfer in a continuous galvanizing bath. In: Proceedings 17th AIAA Computational Fluid Dynamics Conference; 2005 June 6-9; Toronto. Proceedings. Reston, VA: AIAA. p. 1-15.

4. Park JH, Park GH, Paik DJ, Huh Y, Hong MH. Influence of aluminum on the formation behavior of Zn-Al-Fe Intermetallic particles in a Zinc Bath. Metall Mater Trans, A Phys Metall Mater Sci. 2012;43A:195-206.

5. Dash SK, Dutta M, Rajesh N. Numerical analysis of fluid flow and heat transfer in molten zinc pot of continuous hot-dip galvanizing Line. ISIJ Int. 2005;45:1059-65.

6. Ajersch F, Ilinca F, Hétu JF, Goodwin FE. Numerical simulation of flow, temperature and composition variation in a Glvanization Bath. Can Metall Q. 2005;44:369-78.

7. Ilinca F, Ajersch F, Baril C, Goodwin FE. Numerical simulation of the galvanizing process during GA to GI Transition. Int $\mathrm{J}$ Numer Methods Fluids. 2007;53:1629-46.

8. Shih TH, Liou W, Shabbir A, Yang Z, Zhu J. A New k-(Eddy viscosity model for high reynolds number turbulent flows Model development and validation. Comput Fluids, 1994. 24:1-30.

9. Ajersch F, Ilinca F. Review of modeling and simulation of galvanizing operations. Steel Res Int. 2018;89(1):1-12.

10. Ajersch F, Ilinca F, Hétu JF. Simulation of flow in a continuous galvanizing bath: part I. Thermal effects of ingot addition. Metall Mater Trans, B, Process Metall Mater Proc Sci. 2004;35B:16170.

11. Zhou X, Yuan S, Huang Y, Yang P, Zhou X. Simulation of special flow affecting dross formation on steel strip in galvanising bath. Ironmak Steelmak. 2015;42:785-90.

12. Lee SJ, Kim S, Koh MS, Choi J. Flow field analysis inside a Molten $\mathrm{Zn}$ Pot of the Continuous Hot-dip Galvanizing Process. ISIJ Int. 2002;42:407-13.

13. Sun H, Zhi DD, Dong AP. Numerical analysis of two-phase flow and heat transfer in external cyclic purification hot-dip galvanizing bath. In 2nd Annual International Conference on Advance Material Engineering; 2016 Apr 15-17; Wuhan, China. Proceedings. Atlantis Press; 2016. p. 1251-7. 
14. Park HS, Han KA, Lee J, Shim JW. Numerical simulation of zinc flow and temperature distribution in a galvanizing zinc pot. ISIJ Int. 2008;48:224-9.

15. Zhou X, Yuan S, Liu C, Yang P, Qian C, Song B. Performance of inductors attached to a galvanizing Bath. Metall Mater Trans, B, Process Metall Mater Proc Sci. 2013;44B:1580-5.

16. Sawaitul P, Chowriwar SA, Lade P. Minimization of dross formation during the continuous galvanizing process in the steel industry. Int J Emerg Technol Adv Eng. 2012;2:45-51.
17. Sippola P, Smith D. Use of high zinc bath entry strip temperature to solve coating problems. Corrosion Science and Technology. 2010;9:175-86.

18. Chen L, Fourmentin R, McDermid JR. Short term formation of the inhibition layer during continuous hot-dip galvanizing. In 7th International Conference on Zinc and Zinc Alloy Coated Steel Sheet Proceedings; 2007 Nov 18-17; Osaka, Japan. Proceedings; 2007. p. 321-6. 